CRYSTALLOGRAPHIC COMMUNICATIONS

ISSN 2056-9890

Received 3 August 2020

Accepted 16 September 2020

Edited by M. Weil, Vienna University of Technology, Austria

Keywords: iron(II) complex; thiocyanate complex; high spin state; trigonal distortion; crystal structure.

CCDC reference: 2032292

Supporting information: this article has supporting information at journals.iucr.org/e

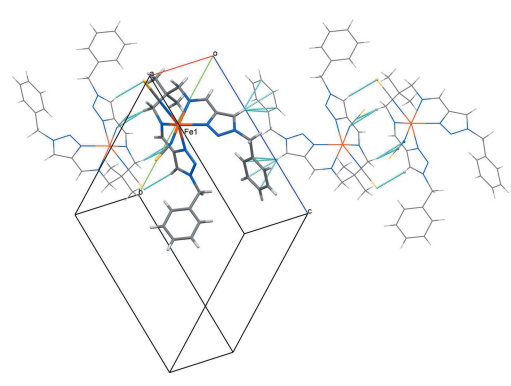

\section{Crystal structure of $\left\{N^{1}, N^{3}-\right.$ bis $[(1-$ benzyl-1H-1,2,3- triazol-4-yl)methylidene]-2,2-dimethylpropane-1,3- diamine\}bis(thiocyanato- $\kappa \mathrm{N}$ )iron(II)}

\author{
Kateryna Znovjyak, ${ }^{a}$ Maksym Seredyuk, ${ }^{\mathrm{a} *}$ Sergey O. Malinkin, ${ }^{\mathrm{a}}$ Sergiu Shova ${ }^{\mathrm{b}}$ and \\ Lutfullo Soliev ${ }^{\mathrm{c} *}$
}

\begin{abstract}
${ }^{a}$ Department of Chemistry, Taras Shevchenko National University of Kyiv, Volodymyrska Street 64, Kyiv, 01601, Ukraine, bepartment of Inorganic Polymers, "Petru Poni" Institute of Macromolecular, Chemistry, Romanian Academy of Science, Aleea Grigore Ghica Voda 41-A, lasi, 700487, Romania, and ' Department of General and Inorganic Chemistry, Faculty of Chemistry, Tajik State Pedagogical University, Rudaki 121, 734003 Dushanbe, Tajikistan. *Correspondence e-mail: mlseredyuk@gmail.com,soliev.lutfullo@yandex.com
\end{abstract}

The unit cell of the title compound, $\left[\mathrm{Fe}^{\mathrm{II}}(\mathrm{NCS})_{2}\left(\mathrm{C}_{25} \mathrm{H}_{28} \mathrm{~N}_{8}\right)\right]$, consists of two charge-neutral complex molecules related by an inversion centre. In the complex molecule, the tetradentate ligand $N^{1}, N^{3}$-bis[(1-benzyl-1H-1,2,3-triazol4-yl)methylene]-2,2-dimethylpropane-1,3-diamine coordinates to the $\mathrm{Fe}^{\mathrm{II}}$ ion through the $\mathrm{N}$ atoms of the 1,2,3-triazole moieties and aldimine groups. Two thiocyanate anions, coordinating through their $\mathrm{N}$ atoms, complete the coordination sphere of the central ion. In the crystal, neighbouring molecules are linked through weak $\mathrm{C}-\mathrm{H} \cdots \pi, \mathrm{C}-\mathrm{H} \cdots \mathrm{S}$ and $\mathrm{C}-\mathrm{H} \cdots \mathrm{N}$ interactions into a two-dimensional network extending parallel to (011). The intermolecular contacts were quantified using Hirshfeld surface analysis and two-dimensional fingerprint plots, revealing the relative contributions of the contacts to the crystal packing to be $\mathrm{H} \cdots \mathrm{H}(35.2 \%), \mathrm{H} \cdots \mathrm{C} / \mathrm{C} \cdots \mathrm{H}(26.4 \%), \mathrm{H} \cdots \mathrm{S} / \mathrm{S} \cdots \mathrm{H}$ $(19.3 \%)$ and $\mathrm{H} \cdots \mathrm{N} / \mathrm{N} \cdots \mathrm{H}(13.9 \%)$.

\section{Chemical context}

Coordination complexes of $3 d$ transition metals represent a large class of potentially applicable materials exhibiting catalytic (Strotmeyer et al., 2003), magnetic (Pavlishchuk et al., 2010) and spin-switching functionalities (Gütlich \& Goodwin, $2004)$ with easily detectable and exploitable variations of physical properties (Gural'skiy et al., 2012; Suleimanov et al., 2015).

Iron(II) complexes based on Schiff bases derived from Nsubstituted 1,2,3-triazole aldehydes represent an interesting class of coordination compounds exhibiting spin-state switching between low- and high-spin states in different temperature regions (Hagiwara et al., 2014, 2016, 2020; Hora \& Hagiwara, 2017). In charge-neutral mononuclear complexes of this kind described so far, the thiocyanate anions occupy the axial position of the coordination sphere and thus are in a trans-configuration (Hagiwara \& Okada, 2016; Hagiwara et al., 2017).

Having ongoing interest in functional $3 d$ metal complexes formed by polydentate ligands (Seredyuk et al., 2006, 2007, 2011, 2015, 2016; Seredyuk, 2012; Valverde-Muñoz et al., 2020), we report here the synthesis and crystal structure of a new high-spin $\mathrm{Fe}^{\mathrm{II}}$ complex based on the tetradentate ligand $N^{1}, N^{3}$-bis[(1-benzyl-1H-1,2,3-triazol-4-yl)methylene]-2,2-dimethylpropane-1,3-diamine with thiocyanate anions arranged in a cis-configuration. 


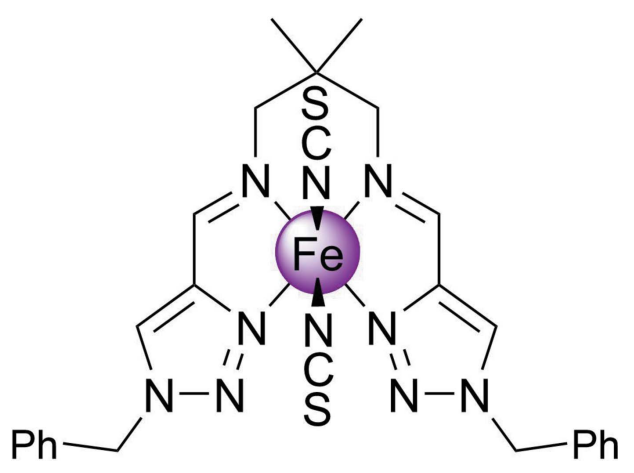

\section{Structural commentary}

The $\mathrm{Fe}^{\mathrm{II}}$ ion of the title complex has a distorted trigonalprismatic $\mathrm{N}_{6}$ coordination environment formed by four $\mathrm{N}$ atoms of the tetradentate Schiff-base ligand and two $\mathrm{NCS}^{-}$ counter-ions (Fig. 1). The average bond length $\langle\mathrm{Fe}-\mathrm{N}\rangle=$ 2.19 (9) $\AA$ is typical for high-spin complexes with an $\left[\mathrm{FeN}_{6}\right]$ chromophore (Gütlich \& Goodwin, 2004). The N$\mathrm{Fe}-\mathrm{N}$ angle between the cis-aligned thiocyanate $\mathrm{N}$ atoms is $87.58(9)^{\circ}$. The average trigonal distortion parameters $\Sigma=\Sigma_{1}^{12}\left(\mid 90-\varphi_{i}\right)$, where $\varphi_{i}$ is the angle $\mathrm{N}-\mathrm{Fe}-\mathrm{N}^{\prime}$ (Drew $e t$ al., 1995), and $\Theta=\Sigma_{1}^{24}\left(\left|60-\theta_{i}\right|\right)$, where $\theta_{i}$ is the angle generated by superposition of two opposite faces of an octahedron (Chang et al., 1990), are 453.2 and $149.38^{\circ}$, respectively. These values reveal a great deviation of the coordination environment from an ideal octahedron (where $\Sigma=\Theta=0$ ), and are significantly larger than those of similar $\left[\mathrm{FeN}_{6}\right]$ high-spin trans-complexes (Hagiwara et al., 2017). With the aid of

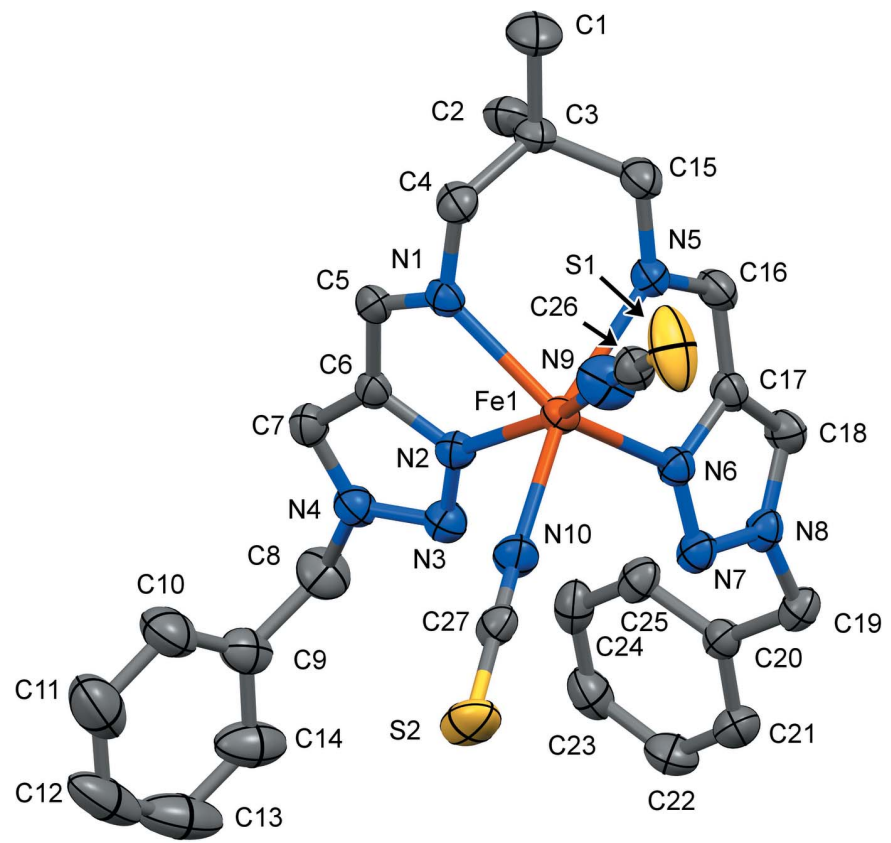

Figure 1

The molecular structure of the title compound with displacement ellipsoids drawn at the $50 \%$ probability level. $\mathrm{H}$ atoms have been omitted for clarity.
Table 1

Hydrogen-bond geometry $\left(\AA,^{\circ}\right)$.

$\mathrm{Cg}$ is the centroid of the $\mathrm{C} 20-\mathrm{C} 25$ ring.

\begin{tabular}{llllll}
\hline$D-\mathrm{H} \cdots A$ & $D-\mathrm{H}$ & $\mathrm{H} \cdots A$ & $D \cdots A$ & $D-\mathrm{H} \cdots A$ \\
\hline $\mathrm{C} 18-\mathrm{H} 18 \cdots C g^{\mathrm{i}}$ & 0.93 & 2.42 & $3.330(3)$ & 167 & \\
$\mathrm{C} 19-\mathrm{H} 19 A \cdots \mathrm{N} 7^{\mathrm{ii}}$ & 0.97 & 2.38 & $3.311(3)$ & 162 & \\
$\mathrm{C} 21-\mathrm{H} 21 \cdots \mathrm{C} 27^{\mathrm{ii}}$ & 0.93 & 2.89 & $3.603(3)$ & 134 & \\
$\mathrm{C} 7-\mathrm{H} 7 \cdots \mathrm{S} 1^{\mathrm{iii}}$ & 0.93 & 2.87 & $3.755(3)$ & 159 & \\
$\mathrm{C} 4-\mathrm{H} 4 B \cdots \mathrm{N} 10^{\mathrm{iii}}$ & 0.97 & 2.69 & $3.617(3)$ & 160 & \\
$\mathrm{C} 4-\mathrm{H} 4 B \cdots \mathrm{C} 27^{\mathrm{iii}}$ & 0.97 & 2.75 & $3.709(3)$ & 171 & \\
\hline Symmetry codes: & (i) & $-x,-y,-z+1 ;$ & (ii) & $-x+1,-y,-z+1 ;$ & (iii) \\
$-x+1,-y+1,-z$. & & & & &
\end{tabular}

continuous shape measure (CShM), the closest shape of a coordination polyhedron and its distortion can be determined numerically (Kershaw Cook et al., 2015). The calculated CShM value relative to the ideal $O_{h}$ symmetry for an octahedron is 6.285 , while it is 4.008 relative to the ideal $D_{3 h}$ symmetry for a trigonal prism. Hence, the polyhedron is closer to the latter shape; however, it is notably distorted (for the ideal polyhedron $\mathrm{CShM}=0$ ). The volume of the $\left[\mathrm{FeN}_{6}\right]$ coordination polyhedron is $12.4 \AA^{3}$.

\section{Supramolecular features}

Neighbouring complex molecules form dimers through double weak contacts $\mathrm{C} 18-\mathrm{H} 18 B \cdots C g^{\mathrm{i}}$ of $3.330(3) \AA$ (Cg corresponds to the centroid of the $\mathrm{C} 20-\mathrm{C} 25$ phenyl ring; symmetry codes refer to Table 1). The $\mathrm{CH}$ group of one of the triazole rings forms a weak hydrogen bond $\mathrm{C} 7-\mathrm{H} 7 \cdots \mathrm{S} 1^{\text {ii }}$ [3.755 (3) $\AA$ ] with a thiocyanate anion. This, together with the $\mathrm{C} 4-\mathrm{H} 4 B \cdots \mathrm{C} 27^{\mathrm{ii}}$ and $\mathrm{C} 4-\mathrm{H} 4 B \cdots \mathrm{N} 10^{\mathrm{ii}}$ interactions [3.709 (3) and 3.617 (3) $\AA$ ] involving the $\mathrm{C} \equiv \mathrm{N}$ group of the anion, links the dimers into a supramolecular chain propagating parallel to [011] (Fig. 2). These chains are weakly bound through double contacts between the benzyl groups and the thiocyanate anions $\left[\mathrm{C} 21-\mathrm{H} 21 \cdots \mathrm{C} 27^{\mathrm{iii}}=3.603(3) \AA\right]$ and triazole groups $\left[\mathrm{C} 19-\mathrm{H} 19 A \cdots \mathrm{N} 7^{\mathrm{iii}}=3.311(3) \AA\right]$ of neigh-

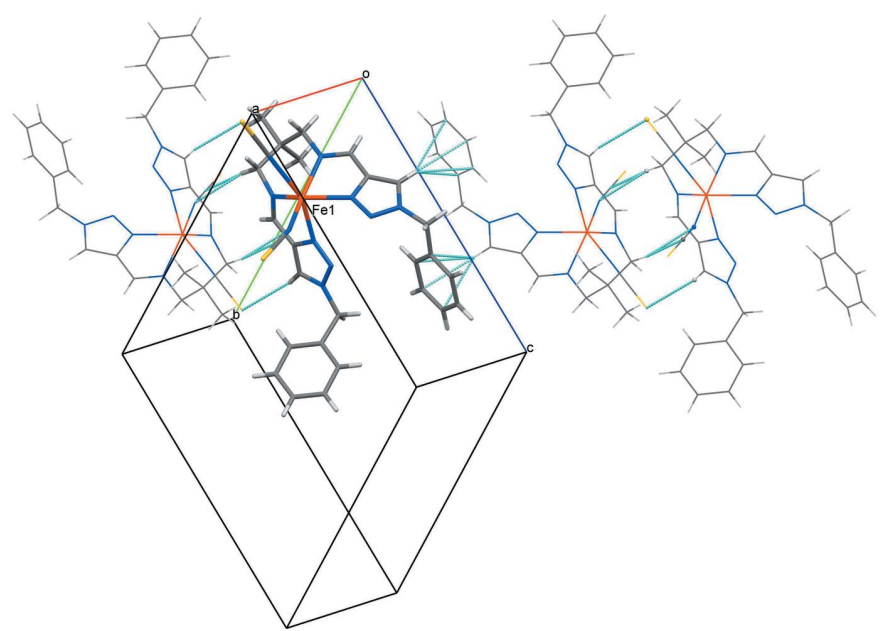

Figure 2

Weak hydrogen bonding (cyan dashed lines), resulting in the formation of chains in the packing. 

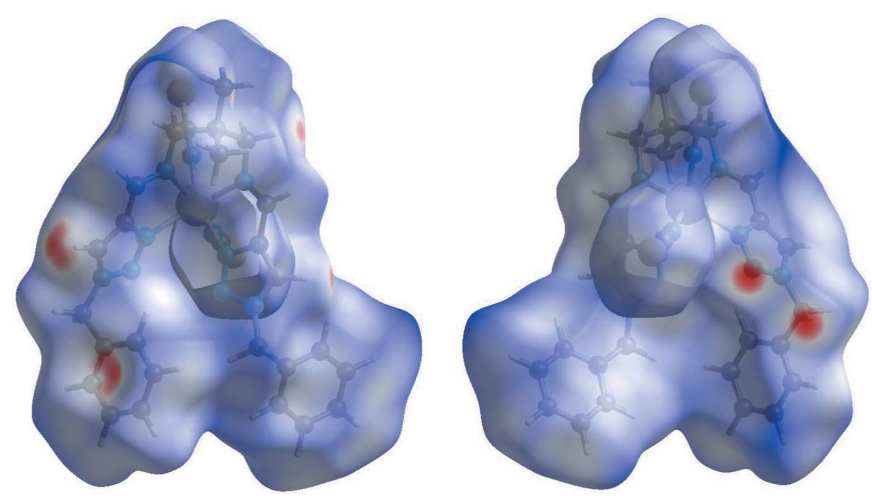

Figure 3

Two projections of $d_{\text {norm }}$ mapped on Hirshfeld surfaces, showing the intermolecular interactions within the molecule. Red areas represent contacts shorter than the sum of the van der Waals radii, while blue areas represent regions where contacts are larger than the sum of van der Waals radii, and white areas are zones close to the sum of van der Waals radii.

(a)
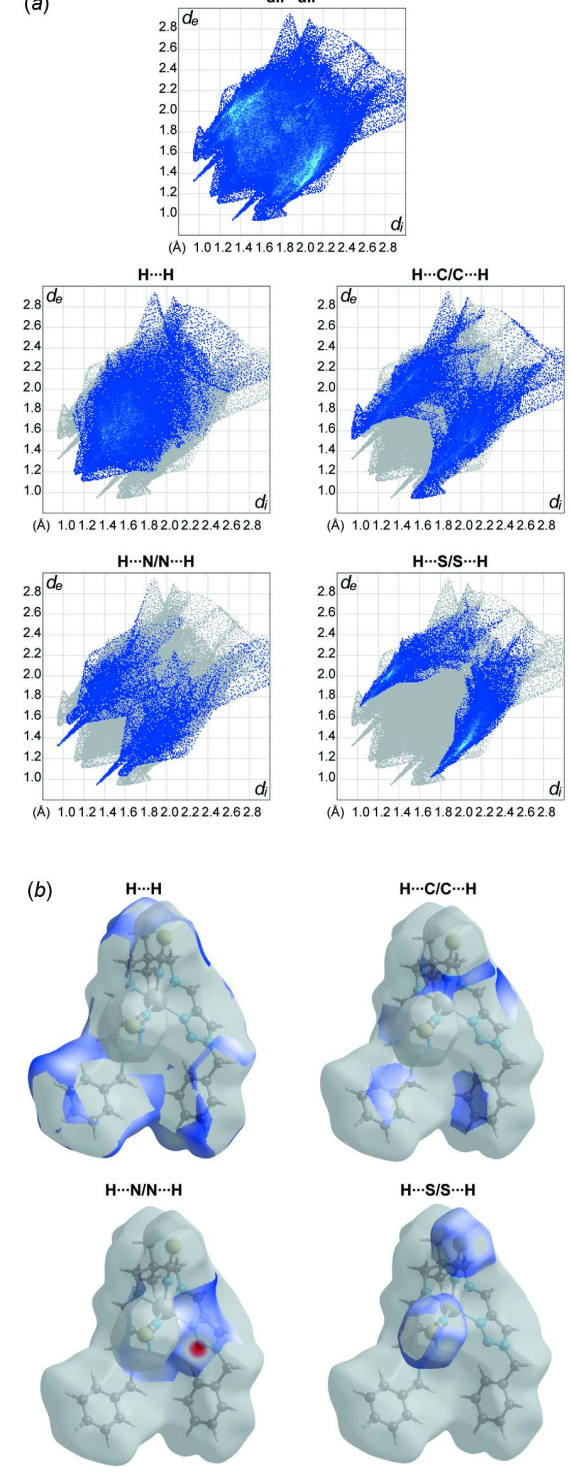

Figure 4

(a) The overall two-dimensional fingerprint plot and those decomposed into specified interactions. (b) Hirshfeld surface representations with the function $d_{\text {norm }}$ plotted onto the surface for the different interactions.
Table 2

Comparison of the distortion parameters for indicated $\mathrm{Fe}^{\mathrm{II}}$ complexes.

Parameters for OTANOO averaged over five independent complex cations.

\begin{tabular}{lllll}
\hline Compound & $<\mathrm{Fe}-\mathrm{N}>(\AA)$ & $\Sigma\left(^{\circ}\right)$ & $\Theta\left({ }^{\circ}\right)$ & $\operatorname{CShM}\left(D_{3 h}\right)$ \\
\hline Title compound & 2.186 & 453.2 & 149.38 & 4.008 \\
CABLOH & 1.899 & 725.74 & 178.16 & 0.525 \\
BUNSAF & 2.218 & 703.65 & 201.07 & 1.887 \\
OWIHAE & 2.202 & 894.48 & 206.57 & 0.602 \\
OTANOO & 2.191 & 697.3 & 183.24 & 1.098 \\
\hline
\end{tabular}

bouring complex molecules, forming a two-dimensional supramolecular array extending parallel to (011).

\section{Hirshfeld surface and 2D fingerprint plots}

Hirshfeld surface analysis was performed and the associated two-dimensional fingerprint plots were generated using Crystal Explorer (Turner et al., 2018), with a standard resolution of the three-dimensional $d_{\text {norm }}$ surfaces plotted over a fixed colour scale of -0.2801 (red) to 1.8236 (blue) a.u. The pale-red spots symbolize short contacts and negative $d_{\text {norm }}$ values on the surface correspond to the interactions described above. The overall two-dimensional fingerprint plot is illustrated in Fig. 3. The Hirshfeld surfaces mapped over $d_{\text {norm }}$ are shown for the $\mathrm{H} \cdots \mathrm{H}, \mathrm{H} \cdots \mathrm{C} / \mathrm{C} \cdots \mathrm{H}, \mathrm{H} \cdots \mathrm{S} / \mathrm{S} \cdots \mathrm{H}$, and $\mathrm{H} \cdots \mathrm{N} /$ $\mathrm{N} \cdots \mathrm{H}$ contacts, and the two-dimensional fingerprint plots are presented in Fig. 4, associated with their relative contributions to the Hirshfeld surface. At $35.2 \%$, the largest contribution to the overall crystal packing is from $\mathrm{H} \cdots \mathrm{H}$ interactions, which are located in the middle region of the fingerprint plot. $\mathrm{H} \cdots \mathrm{C} /$ $\mathrm{C} \cdots \mathrm{H}$ contacts contribute $26.4 \%$, and the $\mathrm{H} \cdots \mathrm{S} / \mathrm{S} \cdots \mathrm{H}$ contacts contribute $19.3 \%$ to the Hirshfeld surface, both resulting in a pair of characteristic wings. The $\mathrm{H} \cdots \mathrm{N} / \mathrm{N} \cdots \mathrm{H}$ contacts, represented by a pair of sharp spikes in the fingerprint plot, make a $13.9 \%$ contribution to the Hirshfeld surface.

\section{Database survey}

A search of the Cambridge Structural Database (CSD 2020, update of May 2020; Groom et al., 2016) revealed four similar $\mathrm{Fe}^{\mathrm{II}}$ thiocyanate complexes, derivatives of a 1,3-diaminopropanes and $N$-substituted 1,2,3-triazole aldehydes, viz. DURXEV, ADAQUU, ADAREF and solvatomorphs ADAROP and ADARUV (Hagiwara et al., 2017; Hagiwara \& Okada, 2016). These complexes show hysteretic spin crossover with the $\mathrm{Fe}-\mathrm{N}$ distances in the range 1.931-1.959 $\AA$ for the low-spin state and 2.154-2.169 $\AA$ for the high-spin state of the $\mathrm{Fe}^{\mathrm{II}}$ ions. The reported pseudo-trigonal-prismatic complexes with an $\left[\mathrm{FeN}_{6}\right]$ chromophore are formed by structurally hindered rigid hexadentate ligands favoring a trigonal-prismatic environment of the central $\mathrm{Fe}^{\mathrm{II}}$ ion in the low- or highspin state: CABLOH (Voloshin et al., 2001), BUNSAF (El Hajj et al., 2009), OWIHAE (Seredyuk et al., 2011), OTANOO (Stock et al., 2016). For comparison purposes, Table 2 collates the distortion parameters $\Sigma, \Theta$ and CShM for the latter complexes. 


\section{Synthesis and crystallization}

The ligand of the title compound was obtained in situ by condensation of 1 eq. of 2,2-dimethyl-1,3-propanediamine with 2.2 eq. of 1-benzyl-1H-1,2,3-triazole-4-carbaldehyde in boiling methanol over $5 \mathrm{~min}$ and subsequent reaction with 1 eq. of $\left[\mathrm{Fe}(\mathrm{py})_{4}(\mathrm{NCS})_{2}\right]$ dissolved in a minimum amount of boiling methanol with a minimum amount of ascorbic acid. The formed yellow solution was slowly cooled to ambient temperature. The formed orange crystals were subsequently filtered off. Elemental analysis calculated (\%) for $\mathrm{C}_{27} \mathrm{H}_{28} \mathrm{FeN}_{10} \mathrm{~S}_{2}$ : C, 52.94; H, 4.61; N, 22.87; S, 10.47; found: C, $52.88 ; \mathrm{H}, 4.37 ; \mathrm{N}, 22.40 ; \mathrm{S}, 10.35$. IR $v \mathrm{KBr}\left(\mathrm{cm}^{-1}\right): 1615$ $(\mathrm{C}=\mathrm{N}), 2071,2115$ (NCS).

\section{Refinement}

Crystal data, data collection and structure refinement details are summarized in Table 3. $\mathrm{H}$ atoms were placed in calculated positions using idealized geometries, with $\mathrm{C}-\mathrm{H}=0.96-0.97 \AA$ for methylene and methyl groups and $0.93 \AA$ for aromatic $\mathrm{H}$ atoms, and refined using a riding model with $U_{\text {iso }}(\mathrm{H})=1.2-$ $1.5 U_{\text {eq }}(\mathrm{C})$.

\section{Funding information}

Funding for this research was provided by: H2020 Marie Skłodowska-Curie Actions (grant No. 734322).

\section{References}

Bourhis, L. J., Dolomanov, O. V., Gildea, R. J., Howard, J. A. K. \& Puschmann, H. (2015). Acta Cryst. A71, 59-75.

Chang, H. R., McCusker, J. K., Toftlund, H., Wilson, S. R., Trautwein, A. X., Winkler, H. \& Hendrickson, D. N. (1990). J. Am. Chem. Soc. 112, 6814-6827.

Dolomanov, O. V., Bourhis, L. J., Gildea, R. J., Howard, J. A. K. \& Puschmann, H. (2009). J. Appl. Cryst. 42, 339-341.

Drew, M. G. B., Harding, C. J., McKee, V., Morgan, G. G. \& Nelson, J. (1995). J. Chem. Soc. Chem. Commun. pp. 1035-1038.

El Hajj, F., Sebki, G., Patinec, V., Marchivie, M., Triki, S., Handel, H., Yefsah, S., Tripier, R., Gómez-García, C. J. \& Coronado, E. (2009). Inorg. Chem. 48, 10416-10423.

Groom, C. R., Bruno, I. J., Lightfoot, M. P. \& Ward, S. C. (2016). Acta Cryst. B72, 171-179.

Gural'skiy, I. A., Quintero, C. M., Molnár, G., Fritsky, I. O., Salmon, L. \& Bousseksou, A. (2012). Chem. Eur. J. 18, 9946-9954.

Gütlich, P. \& Goodwin, H. A. (2004). Top. Curr. Chem. 233, 1-47.

Hagiwara, H., Masuda, T., Ohno, T., Suzuki, M., Udagawa, T. \& Murai, K.-I. (2017). Cryst. Growth Des. 17, 6006-6019.

Hagiwara, H., Minoura, R., Okada, S. \& Sunatsuki, Y. (2014). Chem. Lett. 43, 950-952.

Hagiwara, H., Minoura, R., Udagawa, T., Mibu, K. \& Okabayashi, J. (2020). Inorg. Chem. 59, 9866-9880.

Hagiwara, H. \& Okada, S. (2016). Chem. Commun. 52, 815-818.

Hagiwara, H., Tanaka, T. \& Hora, S. (2016). Dalton Trans. 45, 1713217140.

Hora, S. \& Hagiwara, H. (2017). Inorganics, 5, 49.

Kershaw Cook, L. J., Mohammed, R., Sherborne, G., Roberts, T. D., Alvarez, S. \& Halcrow, M. A. (2015). Coord. Chem. Rev. 289-290, 2-12.

Pavlishchuk, A. V., Kolotilov, S. V., Zeller, M., Thompson, L. K., Fritsky, I. O., Addison, A. W. \& Hunter, A. D. (2010). Eur. J. Inorg. Chem. pp. 4851-4858.
Table 3

Experimental details.

\begin{tabular}{|c|c|}
\hline \multicolumn{2}{|l|}{ Crystal data } \\
\hline Chemical formula & {$\left[\mathrm{Fe}(\mathrm{NCS})_{2}\left(\mathrm{C}_{25} \mathrm{H}_{28} \mathrm{~N}_{8}\right)\right]$} \\
\hline$M_{\mathrm{r}}$ & 612.56 \\
\hline Crystal system, space group & Triclinic, $P \overline{1}$ \\
\hline Temperature $(\mathrm{K})$ & 250 \\
\hline$a, b, c(\AA)$ & $8.9656(5), 12.5060(6), 14.2311$ (7) \\
\hline$\alpha, \beta, \gamma\left({ }^{\circ}\right)$ & $67.552(5), 85.106(4), 84.087(4)$ \\
\hline$V\left(\AA^{3}\right)$ & $1465.06(14)$ \\
\hline$Z$ & 2 \\
\hline Radiation type & Мо $K \alpha$ \\
\hline$\mu\left(\mathrm{mm}^{-1}\right)$ & 0.69 \\
\hline Crystal size $(\mathrm{mm})$ & $0.4 \times 0.2 \times 0.2$ \\
\hline \multicolumn{2}{|l|}{ Data collection } \\
\hline Diffractometer & $\begin{array}{l}\text { Rigaku Oxford Diffraction } \\
\text { Xcalibur, Eos }\end{array}$ \\
\hline Absorption correction & $\begin{array}{l}\text { Multi-scan (CrysAlis PRO; Rigaku } \\
\text { OD, 2018) }\end{array}$ \\
\hline$T_{\min }, T_{\max }$ & $0.911,1.000$ \\
\hline $\begin{array}{l}\text { No. of measured, independent and } \\
\text { observed }[I>2 \sigma(I)] \text { reflections }\end{array}$ & $10677,5175,4416$ \\
\hline$R_{\text {int }}$ & 0.018 \\
\hline$(\sin \theta / \lambda)_{\max }\left(\AA^{-1}\right)$ & 0.595 \\
\hline \multicolumn{2}{|l|}{ Refinement } \\
\hline$R\left[F^{2}>2 \sigma\left(F^{2}\right)\right], w R\left(F^{2}\right), S$ & $0.037,0.082,1.03$ \\
\hline No. of reflections & 5175 \\
\hline No. of parameters & 391 \\
\hline $\mathrm{H}$-atom treatment & $\begin{array}{l}\text { Only H-atom displacement para- } \\
\text { meters refined }\end{array}$ \\
\hline$\Delta \rho_{\max }, \Delta \rho_{\min }\left(\mathrm{e} \AA^{-3}\right)$ & $0.62,-0.59$ \\
\hline
\end{tabular}

Computer programs: CrysAlis PRO (Rigaku OD, 2018), olex2.solve (Bourhis et al., 2015), SHELXL2018/3 (Sheldrick, 2015) and OLEX2 (Dolomanov et al., 2009).

Rigaku OD (2018). CrysAlis PRO. Rigaku Oxford Diffraction, Yarnton, England.

Seredyuk, M. (2012). Inorg. Chim. Acta, 380, 65-71.

Seredyuk, M., Gaspar, A. B., Ksenofontov, V., Reiman, S., Galyametdinov, Y., Haase, W., Rentschler, E. \& Gütlich, P. (2006). Hyperfine Interact. 166, 385-390.

Seredyuk, M., Gaspar, A. B., Kusz, J. \& Gütlich, P. (2011). Z. Anorg. Allg. Chem. 637, 965-976.

Seredyuk, M., Haukka, M., Fritsky, I. O., Kozłowski, H., Krämer, R., Pavlenko, V. A. \& Gütlich, P. (2007). Dalton Trans. pp. 31833194.

Seredyuk, M., Piñeiro-López, L., Muñoz, M. C., Martínez-Casado, F. J., Molnár, G., Rodriguez-Velamazán, J. A., Bousseksou, A. \& Real, J. A. (2015). Inorg. Chem. 54, 7424-7432.

Seredyuk, M., Znovjyak, K., Muñoz, M. C., Galyametdinov, Y., Fritsky, I. O. \& Real, J. A. (2016). RSC Adv. 6, 39627-39635.

Sheldrick, G. M. (2015). Acta Cryst. C71, 3-8.

Stock, P., Deck, E., Hohnstein, S., Korzekwa, J., Meyer, K., Heinemann, F. W., Breher, F. \& Hörner, G. (2016). Inorg. Chem. 55, 5254-5265.

Strotmeyer, K. P., Fritsky, I. O., Ott, R., Pritzkow, H. \& Krämer, R. (2003). Supramol. Chem. 15, 529-547.

Suleimanov, I., Kraieva, O., Costa, J. S., Fritsky, I. O., Molnár, G., Salmon, L. \& Bousseksou, A. (2015). J. Mater. Chem. C. 3, 50265032.

Turner, M. J., Mckinnon, J. J., Wolff, S. K., Grimwood, D. J., Spackman, P. R., Jayatilaka, D. \& Spackman, M. A. (2018). CrystalExplorer 17.5. The University of Western Australia.

Valverde-Muñoz, F. J., Seredyuk, M., Muñoz, M. C., Molnár, G., Bibik, Y. S. \& Real, J. A. (2020). Angew. Chem., Int. Ed. https:// doi.org/10.1002anie.202006453

Voloshin, Y. Z., Varzatskii, O. A., Stash, A. I., Belsky, V. K., Bubnov, Y. N., Vorontsov, I. I., Potekhin, K. A., Polshin, E. V. \& Antipin, M. Y. (2001). Polyhedron, 20, 2721-2733. 


\section{supporting information}

Acta Cryst. (2020). E76, 1661-1664 [https://doi.org/10.1107/S2056989020012608]

Crystal structure of $\left\{N^{1}, N^{3}\right.$-bis [(1-benzyl-1H-1,2,3-triazol-4-yl)methylidene]-2,2-dimethylpropane-1,3-diamine\}bis(thiocyanato- $\kappa$ N)iron(II)

Kateryna Znovjyak, Maksym Seredyuk, Sergey O. Malinkin, Sergiu Shova and Lutfullo Soliev

Computing details

Data collection: CrysAlis PRO (Rigaku OD, 2018); cell refinement: CrysAlis PRO (Rigaku OD, 2018); data reduction: CrysAlis PRO (Rigaku OD, 2018); program(s) used to solve structure: olex2.solve (Bourhis et al., 2015); program(s) used to refine structure: SHELXL2018/3 (Sheldrick, 2015); molecular graphics: OLEX2 (Dolomanov et al., 2009); software used to prepare material for publication: OLEX2 (Dolomanov et al., 2009).

$\left\{N^{1}, N^{3}\right.$-Bis [(1-benzyl-1H-1,2,3-triazol-4-yl)methylidene]-2,2-dimethylpropane-1,3-diamine $\}$ bis(thiocyanato$\kappa N) \operatorname{iron}(\mathrm{II})$

Crystal data

$\left[\mathrm{Fe}(\mathrm{NCS})_{2}\left(\mathrm{C}_{25} \mathrm{H}_{28} \mathrm{~N}_{8}\right)\right]$

$Z=2$

$M_{r}=612.56$

$F(000)=636$

Triclinic, $P \overline{1}$

$D_{\mathrm{x}}=1.389 \mathrm{Mg} \mathrm{m}^{-3}$

$a=8.9656(5) \AA$

$b=12.5060(6) \AA$

$c=14.2311(7) \AA$

$\alpha=67.552(5)^{\circ}$

$\beta=85.106(4)^{\circ}$

$\gamma=84.087(4)^{\circ}$

$V=1465.06(14) \AA^{3}$

Mo $K \alpha$ radiation, $\lambda=0.71073 \AA$

Cell parameters from 4582 reflections

$\theta=1.6-28.8^{\circ}$

$\mu=0.69 \mathrm{~mm}^{-1}$

$T=250 \mathrm{~K}$

Plate, orange

$0.4 \times 0.2 \times 0.2 \mathrm{~mm}$

Data collection

Rigaku Oxford Diffraction Xcalibur, Eos diffractometer

Radiation source: fine-focus sealed X-ray tube, Enhance (Mo) X-ray Source

Graphite monochromator

Detector resolution: 16.1593 pixels $\mathrm{mm}^{-1}$

$\omega$ scans

Absorption correction: multi-scan

(CrysAlisPro; Rigaku OD, 2018)

$T_{\min }=0.911, T_{\max }=1.000$

10677 measured reflections

5175 independent reflections

4416 reflections with $I>2 \sigma(I)$

$R_{\text {int }}=0.018$

$\theta_{\text {max }}=25.0^{\circ}, \theta_{\min }=1.6^{\circ}$

$h=-10 \rightarrow 9$

$k=-14 \rightarrow 13$

$l=-16 \rightarrow 16$

\section{Refinement}

Refinement on $F^{2}$

Least-squares matrix: full

$R\left[F^{2}>2 \sigma\left(F^{2}\right)\right]=0.037$

$w R\left(F^{2}\right)=0.082$

$S=1.03$

5175 reflections

391 parameters

0 restraints

Hydrogen site location: inferred from neighbouring sites

Only H-atom displacement parameters refined 
$w=1 /\left[\sigma^{2}\left(F_{\mathrm{o}}^{2}\right)+(0.0224 P)^{2}+1.1951 P\right]$

where $P=\left(F_{\mathrm{o}}^{2}+2 F_{\mathrm{c}}^{2}\right) / 3$

$(\Delta / \sigma)_{\max }<0.001$

$$
\Delta \rho_{\max }=0.62 \mathrm{e} \AA^{-3}
$$

$\Delta \rho_{\min }=-0.59$ e $\AA^{-3}$

\section{Special details}

Geometry. All esds (except the esd in the dihedral angle between two 1.s. planes) are estimated using the full covariance matrix. The cell esds are taken into account individually in the estimation of esds in distances, angles and torsion angles; correlations between esds in cell parameters are only used when they are defined by crystal symmetry. An approximate (isotropic) treatment of cell esds is used for estimating esds involving 1.s. planes.

Fractional atomic coordinates and isotropic or equivalent isotropic displacement parameters $\left(\hat{A}^{2}\right)$

\begin{tabular}{|c|c|c|c|c|}
\hline & $x$ & $y$ & $z$ & $U_{\text {iso }} * / U_{\text {eq }}$ \\
\hline $\mathrm{Fe} 1$ & $0.46967(4)$ & $0.25650(3)$ & $0.15072(2)$ & $0.02848(10)$ \\
\hline $\mathrm{S} 1$ & $0.83937(11)$ & $0.13105(7)$ & $-0.06302(8)$ & $0.0749(3)$ \\
\hline $\mathrm{S} 2$ & $0.93628(8)$ & $0.30840(7)$ & $0.26836(6)$ & $0.0597(2)$ \\
\hline N1 & $0.3705(2)$ & $0.40653(16)$ & $0.02733(14)$ & $0.0288(4)$ \\
\hline N2 & $0.3331(2)$ & $0.37376(16)$ & $0.22397(14)$ & $0.0307(4)$ \\
\hline N3 & $0.3079(2)$ & 0.37587 (17) & $0.31494(15)$ & $0.0371(5)$ \\
\hline N4 & $0.2416(2)$ & $0.48269(17)$ & $0.30170(15)$ & $0.0357(5)$ \\
\hline N5 & $0.3032(2)$ & $0.17554(16)$ & $0.09218(14)$ & $0.0310(4)$ \\
\hline N6 & $0.3792(2)$ & $0.11839(15)$ & $0.28389(14)$ & $0.0296(4)$ \\
\hline N7 & $0.3924(2)$ & $0.08273(16)$ & $0.38237(15)$ & $0.0331(5)$ \\
\hline N8 & $0.2832(2)$ & $0.01043(15)$ & $0.42600(14)$ & $0.0306(4)$ \\
\hline N9 & $0.6253(3)$ & $0.2060(2)$ & 0.05480 (19) & $0.0528(6)$ \\
\hline N10 & $0.6514(2)$ & $0.28477(17)$ & $0.21860(16)$ & $0.0385(5)$ \\
\hline $\mathrm{C} 1$ & 0.2253 & $0.3438(3)$ & $-0.1867(2)$ & $0.0555(8)$ \\
\hline H1A & 0.193892 & 0.423117 & -0.225831 & $0.056(9)^{*}$ \\
\hline H1B & 0.152987 & 0.294205 & -0.190475 & $0.070(10)^{*}$ \\
\hline $\mathrm{H} 1 \mathrm{C}$ & 0.321299 & 0.323624 & -0.213362 & $0.070(10)^{*}$ \\
\hline $\mathrm{C} 2$ & 0.0854 & $0.3642(2)$ & $-0.0346(2)$ & $0.0419(6)$ \\
\hline $\mathrm{H} 2 \mathrm{~A}$ & 0.091918 & 0.352941 & 0.035621 & $0.049(8)^{*}$ \\
\hline $\mathrm{H} 2 \mathrm{~B}$ & 0.010603 & 0.317602 & -0.040245 & $0.053(8)^{*}$ \\
\hline $\mathrm{H} 2 \mathrm{C}$ & 0.058264 & 0.444495 & -0.073289 & $0.058(9)^{*}$ \\
\hline $\mathrm{C} 3$ & $0.2375(3)$ & $0.3284(2)$ & $-0.07558(17)$ & $0.0341(5)$ \\
\hline $\mathrm{C} 4$ & $0.3598(3)$ & $0.4059(2)$ & $-0.07427(17)$ & $0.0346(6)$ \\
\hline $\mathrm{H} 4 \mathrm{~A}$ & 0.455840 & 0.377726 & -0.096377 & $0.029(6)^{*}$ \\
\hline $\mathrm{H} 4 \mathrm{~B}$ & 0.336486 & 0.484535 & -0.121707 & $0.038(7)^{*}$ \\
\hline $\mathrm{C} 5$ & $0.2984(3)$ & $0.48861(19)$ & $0.04771(18)$ & $0.0307(5)$ \\
\hline H5 & 0.256242 & 0.553702 & -0.003344 & $0.033(6)^{*}$ \\
\hline C6 & $0.2840(2)$ & $0.47738(18)$ & $0.15301(17)$ & $0.0290(5)$ \\
\hline $\mathrm{C} 7$ & $0.2245(3)$ & $0.5470(2)$ & 0.20293 (19) & $0.0351(6)$ \\
\hline $\mathrm{H} 7$ & 0.181423 & 0.622568 & 0.174611 & $0.031(6)^{*}$ \\
\hline $\mathrm{C} 8$ & $0.1953(3)$ & $0.5106(3)$ & $0.3915(2)$ & $0.0493(7)$ \\
\hline H8A & 0.190249 & 0.438538 & 0.450396 & $0.066(9)^{*}$ \\
\hline H8B & 0.094871 & 0.548892 & 0.382656 & $0.067(10)^{*}$ \\
\hline C9 & 0.2961 & $0.5868(2)$ & $0.4139(2)$ & $0.0463(7)$ \\
\hline $\mathrm{C} 10$ & $0.3637(4)$ & $0.6766(3)$ & $0.3392(3)$ & $0.0616(8)$ \\
\hline H10 & 0.351491 & 0.691169 & 0.271088 & $0.064(9)^{*}$ \\
\hline
\end{tabular}




$\begin{array}{lllll}\text { C11 } & 0.4509(4) & 0.7463(3) & 0.3655(4) & 0.0805(11) \\ \text { H11 } & 0.497489 & 0.806878 & 0.314888 & 0.080(12)^{*} \\ \text { C12 } & 0.4680(4) & 0.7252(4) & 0.4664(4) & 0.0851(13) \\ \text { H12 } & 0.527762 & 0.770575 & 0.483973 & 0.106(14)^{*} \\ \text { C13 } & 0.3976(4) & 0.6382(5) & 0.5402(4) & 0.0874(13) \\ \text { H13 } & 0.406323 & 0.625738 & 0.608290 & 0.104(14)^{*} \\ \text { C14 } & 0.3135(4) & 0.5684(4) & 0.5147(3) & 0.0666(10) \\ \text { H14 } & 0.267676 & 0.507861 & 0.565894 & 0.104(15)^{*} \\ \text { C15 } & 0.2819(3) & 0.2003(2) & -0.01518(18) & 0.0373(6) \\ \text { H15A } & 0.204471 & 0.153918 & -0.019696 & 0.042(7)^{*} \\ \text { H15B } & 0.374371 & 0.177465 & -0.045658 & 0.038(7)^{*} \\ \text { C16 } & 0.2287(3) & 0.0988(2) & 0.15956(18) & 0.0364(6) \\ \text { H16 } & 0.155892 & 0.062534 & 0.142359 & 0.048(8)^{*} \\ \text { C17 } & 0.2622(3) & 0.07019(19) & 0.26449(18) & 0.0314(5) \\ \text { C18 } & 0.2011(3) & 0.0009(2) & 0.35577(18) & 0.0357(6) \\ \text { H18 } & 0.119557 & -0.043570 & 0.366906 & 0.042(7)^{*} \\ \text { C19 } & 0.2629(3) & -0.0405(2) & 0.53669(17) & 0.0348(6) \\ \text { H19A } & 0.359759 & -0.070943 & 0.565445 & 0.038(7)^{*} \\ \text { H19B } & 0.200022 & -0.104871 & 0.555534 & 0.019(5)^{*} \\ \text { C20 } & 0.1924(2) & 0.04492(19) & 0.58170(17) & 0.0306(5) \\ \text { C21 } & 0.1961(3) & 0.0160(2) & 0.6857(2) & 0.0419(6) \\ \text { H21 } & 0.247770 & -0.052834 & 0.725248 & 0.049(8)^{*} \\ \text { C22 } & 0.1244(3) & 0.0879(2) & 0.7316(2) & 0.0506(7) \\ \text { H22 } & 0.127846 & 0.067512 & 0.801438 & 0.053(8)^{*} \\ \text { C23 } & 0.0476(3) & 0.1901(2) & 0.6733(2) & 0.0490(7) \\ \text { H23 } & -0.002501 & 0.238136 & 0.704015 & 0.049(8)^{*} \\ \text { C24 } & 0.0450(3) & 0.2211(2) & 0.5698(2) & 0.0427(6) \\ \text { H24 } & -0.005800 & 0.290501 & 0.530513 & 0.048(8)^{*} \\ \text { C25 } & 0.1175(3) & 0.1496(2) & 0.52394(19) & 0.0359(6) \\ \text { H25 } & 0.116296 & 0.171620 & 0.453750 & 0.037(7)^{*} \\ \text { C26 } & 0.7137(3) & 0.1742(2) & 0.00625(19) & 0.0368(6) \\ \text { C27 } & 0.7700(3) & 0.2947(2) & 0.23968(18) & 0.0345(6) \\ & & & & \end{array}$

Atomic displacement parameters $\left(\AA^{2}\right)$

\begin{tabular}{lllllll}
\hline & $U^{11}$ & $U^{22}$ & $U^{33}$ & $U^{12}$ & $U^{13}$ & $U^{23}$ \\
\hline Fe1 & $0.02404(18)$ & $0.02930(18)$ & $0.03075(19)$ & $-0.00346(13)$ & $-0.00173(13)$ & $-0.00939(14)$ \\
S1 & $0.0808(6)$ & $0.0533(5)$ & $0.1010(7)$ & $-0.0154(4)$ & $0.0440(5)$ & $-0.0481(5)$ \\
S2 & $0.0373(4)$ & $0.0653(5)$ & $0.0640(5)$ & $-0.0146(3)$ & $-0.0200(3)$ & $-0.0040(4)$ \\
N1 & $0.0271(10)$ & $0.0298(10)$ & $0.0312(10)$ & $-0.0087(8)$ & $-0.0005(8)$ & $-0.0121(9)$ \\
N2 & $0.0294(11)$ & $0.0304(10)$ & $0.0334(11)$ & $-0.0020(8)$ & $-0.0054(8)$ & $-0.0124(9)$ \\
N3 & $0.0412(12)$ & $0.0357(11)$ & $0.0358(12)$ & $-0.0008(9)$ & $-0.0053(9)$ & $-0.0149(9)$ \\
N4 & $0.0365(12)$ & $0.0361(11)$ & $0.0396(12)$ & $-0.0018(9)$ & $-0.0041(9)$ & $-0.0199(10)$ \\
N5 & $0.0353(11)$ & $0.0284(10)$ & $0.0316(11)$ & $-0.0042(8)$ & $-0.0030(8)$ & $-0.0130(9)$ \\
N6 & $0.0276(10)$ & $0.0269(10)$ & $0.0330(11)$ & $-0.0037(8)$ & $-0.0028(8)$ & $-0.0091(9)$ \\
N7 & $0.0296(11)$ & $0.0307(10)$ & $0.0341(11)$ & $-0.0061(8)$ & $-0.0025(8)$ & $-0.0056(9)$ \\
N8 & $0.0280(10)$ & $0.0274(10)$ & $0.0325(11)$ & $-0.0041(8)$ & $0.0008(8)$ & $-0.0068(9)$ \\
N9 & $0.0371(14)$ & $0.0676(16)$ & $0.0621(16)$ & $0.0012(11)$ & $0.0028(12)$ & $-0.0361(14)$
\end{tabular}




\begin{tabular}{|c|c|c|c|c|c|c|}
\hline N10 & $0.0295(12)$ & $0.0374(12)$ & $0.0464(13)$ & $-0.0031(9)$ & $-0.0078(9)$ & $-0.0122(10)$ \\
\hline $\mathrm{C} 1$ & $0.080(2)$ & 0.0589 (19) & $0.0332(15)$ & $-0.0080(17)$ & $-0.0100(15)$ & $-0.0215(15)$ \\
\hline $\mathrm{C} 2$ & $0.0385(15)$ & $0.0481(16)$ & $0.0431(16)$ & $-0.0039(12)$ & $-0.0114(12)$ & $-0.0196(13)$ \\
\hline $\mathrm{C} 3$ & $0.0418(14)$ & $0.0362(13)$ & $0.0270(12)$ & $-0.0057(11)$ & $-0.0060(10)$ & $-0.0133(11)$ \\
\hline $\mathrm{C} 4$ & $0.0412(15)$ & $0.0341(13)$ & $0.0278(12)$ & $-0.0075(11)$ & $0.0019(10)$ & $-0.0104(11)$ \\
\hline $\mathrm{C} 5$ & $0.0321(13)$ & $0.0261(12)$ & $0.0337(13)$ & $-0.0073(10)$ & $-0.0063(10)$ & $-0.0087(10)$ \\
\hline C6 & $0.0257(12)$ & $0.0245(11)$ & $0.0373(13)$ & $-0.0031(9)$ & $-0.0070(10)$ & $-0.0108(10)$ \\
\hline $\mathrm{C} 7$ & $0.0357(14)$ & $0.0291(13)$ & $0.0426(15)$ & $0.0014(10)$ & $-0.0076(11)$ & $-0.0157(11)$ \\
\hline $\mathrm{C} 8$ & $0.0528(18)$ & $0.0564(18)$ & $0.0449(16)$ & $-0.0012(14)$ & $0.0061(13)$ & $-0.0287(15)$ \\
\hline C9 & $0.0437(16)$ & $0.0541(17)$ & $0.0522(17)$ & $0.0130(13)$ & $-0.0100(13)$ & $-0.0351(15)$ \\
\hline $\mathrm{C} 10$ & $0.070(2)$ & $0.067(2)$ & $0.065(2)$ & $-0.0053(17)$ & $-0.0127(17)$ & $-0.0413(18)$ \\
\hline $\mathrm{C} 11$ & $0.074(3)$ & $0.069(2)$ & $0.118(3)$ & $-0.004(2)$ & $-0.019(2)$ & $-0.055(3)$ \\
\hline $\mathrm{C} 12$ & $0.061(2)$ & $0.111(3)$ & $0.132(4)$ & $0.025(2)$ & -0.039 & $-0.101(3)$ \\
\hline C13 & $0.066(3)$ & $0.143(4)$ & $0.090(3)$ & $0.034(3)$ & -0.033 & -0.089 \\
\hline $\mathrm{C} 14$ & $0.060(2)$ & $0.096(3)$ & $0.058(2)$ & $0.0226(19)$ & $-0.0177(17)$ & $-0.049(2)$ \\
\hline $\mathrm{C} 15$ & $0.0471(16)$ & $0.0363(13)$ & $0.0335(13)$ & $-0.0077(11)$ & $-0.0022(11)$ & $-0.0176(11)$ \\
\hline C16 & $0.0400(14)$ & $0.0351(13)$ & $0.0395(14)$ & $-0.0119(11)$ & $-0.0038(11)$ & $-0.0174(12)$ \\
\hline $\mathrm{C} 17$ & $0.0312(13)$ & $0.0275(12)$ & $0.0371(13)$ & $-0.0065(10)$ & $-0.0023(10)$ & $-0.0127(10)$ \\
\hline $\mathrm{C} 18$ & $0.0332(14)$ & $0.0358(13)$ & $0.0375(14)$ & $-0.0137(11)$ & $-0.0005(11)$ & $-0.0107(11)$ \\
\hline C19 & $0.0339(14)$ & $0.0301(13)$ & $0.0334(13)$ & $-0.0023(10)$ & $-0.0007(10)$ & $-0.0044(11)$ \\
\hline $\mathrm{C} 20$ & $0.0259(12)$ & $0.0291(12)$ & $0.0336(13)$ & $-0.0070(9)$ & $-0.0018(10)$ & $-0.0072(10)$ \\
\hline $\mathrm{C} 21$ & $0.0439(16)$ & $0.0387(14)$ & $0.0409(15)$ & $0.0017(12)$ & $-0.0129(12)$ & $-0.0116(12)$ \\
\hline $\mathrm{C} 22$ & $0.0627(19)$ & $0.0567(18)$ & $0.0413(16)$ & $-0.0051(14)$ & $-0.0105(14)$ & $-0.0265(14)$ \\
\hline $\mathrm{C} 23$ & $0.0525(18)$ & $0.0435(16)$ & $0.0619(19)$ & $-0.0059(13)$ & $-0.0020(14)$ & $-0.0317(15)$ \\
\hline $\mathrm{C} 24$ & $0.0394(15)$ & $0.0281(13)$ & $0.0569(18)$ & $-0.0023(11)$ & $-0.0001(12)$ & $-0.0122(13)$ \\
\hline $\mathrm{C} 25$ & $0.0329(14)$ & $0.0325(13)$ & $0.0342(14)$ & $-0.0055(10)$ & $0.0006(10)$ & $-0.0033(11)$ \\
\hline $\mathrm{C} 26$ & $0.0356(14)$ & 0.0337 (13) & $0.0429(15)$ & -0.0039 & $-0.0030(12)$ & $-0.0160(12)$ \\
\hline $\mathrm{C} 27$ & $0.0349(15)$ & $0.0296(12)$ & $0.0315(13)$ & $-0.0023(10)$ & $-0.0025(10)$ & $-0.0028(10)$ \\
\hline
\end{tabular}

Geometric parameters (A, $\left.{ }^{o}\right)$

\begin{tabular}{llll}
\hline $\mathrm{Fe} 1-\mathrm{N} 1$ & $2.1911(19)$ & $\mathrm{C} 5-\mathrm{C} 6$ & $1.446(3)$ \\
$\mathrm{Fe} 1-\mathrm{N} 2$ & $2.306(2)$ & $\mathrm{C} 6-\mathrm{C} 7$ & $1.364(3)$ \\
$\mathrm{Fe} 1-\mathrm{N} 5$ & $2.2618(19)$ & $\mathrm{C} 7-\mathrm{H} 7$ & 0.9300 \\
$\mathrm{Fe} 1-\mathrm{N} 6$ & $2.1817(18)$ & $\mathrm{C} 8-\mathrm{H} 8 \mathrm{~A}$ & 0.9700 \\
$\mathrm{Fe} 1-\mathrm{N} 9$ & $2.088(2)$ & $\mathrm{C} 8-\mathrm{H} 8 \mathrm{~B}$ & $1.511(4)$ \\
$\mathrm{Fe} 1-\mathrm{N} 10$ & $2.088(2)$ & $\mathrm{C} 8-\mathrm{C} 9$ & $1.370(4)$ \\
$\mathrm{S} 1-\mathrm{C} 26$ & $1.620(3)$ & $\mathrm{C} 9-\mathrm{C} 10$ & $1.383(4)$ \\
$\mathrm{S} 2-\mathrm{C} 27$ & $1.621(3)$ & $\mathrm{C} 9-\mathrm{C} 14$ & 0.9300 \\
$\mathrm{~N} 1-\mathrm{C} 4$ & $1.460(3)$ & $\mathrm{C} 10-\mathrm{H} 10$ & $1.397(4)$ \\
$\mathrm{N} 1-\mathrm{C} 5$ & $1.271(3)$ & $\mathrm{C} 10-\mathrm{C} 11$ & 0.9300 \\
$\mathrm{~N} 2-\mathrm{N} 3$ & $1.305(3)$ & $\mathrm{C} 11-\mathrm{H} 11$ & $1.376(6)$ \\
$\mathrm{N} 2-\mathrm{C} 6$ & $1.361(3)$ & $\mathrm{C} 11-\mathrm{C} 12$ & 0.9300 \\
$\mathrm{~N} 3-\mathrm{N} 4$ & $1.355(3)$ & $\mathrm{C} 12-\mathrm{H} 12$ & $1.357(6)$ \\
$\mathrm{N} 4-\mathrm{C} 7$ & $1.339(3)$ & $\mathrm{C} 12-\mathrm{C} 13$ & 0.9300 \\
$\mathrm{~N} 4-\mathrm{C} 8$ & $1.466(3)$ & $\mathrm{C} 13-\mathrm{H} 13$ & $1.373(5)$ \\
$\mathrm{N} 5-\mathrm{C} 15$ & $1.464(3)$ & $\mathrm{C} 13-\mathrm{C} 14$ & 0.9300 \\
$\mathrm{~N} 5-\mathrm{C} 16$ & $1.267(3)$ & $\mathrm{C} 14-\mathrm{H} 14$ &
\end{tabular}




\begin{tabular}{|c|c|c|c|}
\hline N6-N7 & $1.310(3)$ & $\mathrm{C} 15-\mathrm{H} 15 \mathrm{~A}$ & 0.9700 \\
\hline $\mathrm{N} 6-\mathrm{C} 17$ & $1.359(3)$ & C15-H15B & 0.9700 \\
\hline $\mathrm{N} 7-\mathrm{N} 8$ & $1.345(2)$ & $\mathrm{C} 16-\mathrm{H} 16$ & 0.9300 \\
\hline $\mathrm{N} 8-\mathrm{C} 18$ & $1.338(3)$ & $\mathrm{C} 16-\mathrm{C} 17$ & $1.447(3)$ \\
\hline $\mathrm{N} 8-\mathrm{C} 19$ & $1.459(3)$ & $\mathrm{C} 17-\mathrm{C} 18$ & $1.362(3)$ \\
\hline N9- $\mathrm{C} 26$ & $1.147(3)$ & C18-H18 & 0.9300 \\
\hline $\mathrm{N} 10-\mathrm{C} 27$ & $1.161(3)$ & C19-H19A & 0.9700 \\
\hline $\mathrm{C} 1-\mathrm{H} 1 \mathrm{~A}$ & 0.9600 & C19-H19B & 0.9700 \\
\hline $\mathrm{C} 1-\mathrm{H} 1 \mathrm{~B}$ & 0.9600 & $\mathrm{C} 19-\mathrm{C} 20$ & $1.505(3)$ \\
\hline $\mathrm{C} 1-\mathrm{H} 1 \mathrm{C}$ & 0.9600 & $\mathrm{C} 20-\mathrm{C} 21$ & $1.386(3)$ \\
\hline $\mathrm{C} 1-\mathrm{C} 3$ & $1.530(3)$ & $\mathrm{C} 20-\mathrm{C} 25$ & $1.390(3)$ \\
\hline $\mathrm{C} 2-\mathrm{H} 2 \mathrm{~A}$ & 0.9600 & $\mathrm{C} 21-\mathrm{H} 21$ & 0.9300 \\
\hline $\mathrm{C} 2-\mathrm{H} 2 \mathrm{~B}$ & 0.9600 & $\mathrm{C} 21-\mathrm{C} 22$ & $1.380(4)$ \\
\hline $\mathrm{C} 2-\mathrm{H} 2 \mathrm{C}$ & 0.9600 & $\mathrm{C} 22-\mathrm{H} 22$ & 0.9300 \\
\hline $\mathrm{C} 2-\mathrm{C} 3$ & $1.529(3)$ & $\mathrm{C} 22-\mathrm{C} 23$ & $1.380(4)$ \\
\hline $\mathrm{C} 3-\mathrm{C} 4$ & $1.543(3)$ & $\mathrm{C} 23-\mathrm{H} 23$ & 0.9300 \\
\hline $\mathrm{C} 3-\mathrm{C} 15$ & $1.530(3)$ & $\mathrm{C} 23-\mathrm{C} 24$ & $1.374(4)$ \\
\hline $\mathrm{C} 4-\mathrm{H} 4 \mathrm{~A}$ & 0.9700 & $\mathrm{C} 24-\mathrm{H} 24$ & 0.9300 \\
\hline $\mathrm{C} 4-\mathrm{H} 4 \mathrm{~B}$ & 0.9700 & $\mathrm{C} 24-\mathrm{C} 25$ & $1.379(4)$ \\
\hline $\mathrm{C} 5-\mathrm{H} 5$ & 0.9300 & $\mathrm{C} 25-\mathrm{H} 25$ & 0.9300 \\
\hline $\mathrm{N} 1-\mathrm{Fe} 1-\mathrm{N} 2$ & $72.65(7)$ & $\mathrm{N} 4-\mathrm{C} 7-\mathrm{H} 7$ & 127.6 \\
\hline $\mathrm{N} 1-\mathrm{Fe} 1-\mathrm{N} 5$ & $77.61(7)$ & $\mathrm{C} 6-\mathrm{C} 7-\mathrm{H} 7$ & 127.6 \\
\hline $\mathrm{N} 5-\mathrm{Fe} 1-\mathrm{N} 2$ & $107.15(7)$ & $\mathrm{N} 4-\mathrm{C} 8-\mathrm{H} 8 \mathrm{~A}$ & 108.4 \\
\hline $\mathrm{N} 6-\mathrm{Fe} 1-\mathrm{N} 1$ & $134.53(7)$ & $\mathrm{N} 4-\mathrm{C} 8-\mathrm{H} 8 \mathrm{~B}$ & 108.4 \\
\hline $\mathrm{N} 6-\mathrm{Fe} 1-\mathrm{N} 2$ & $82.74(7)$ & $\mathrm{N} 4-\mathrm{C} 8-\mathrm{C} 9$ & $115.4(2)$ \\
\hline $\mathrm{N} 6-\mathrm{Fe} 1-\mathrm{N} 5$ & $73.95(7)$ & $\mathrm{H} 8 \mathrm{~A}-\mathrm{C} 8-\mathrm{H} 8 \mathrm{~B}$ & 107.5 \\
\hline $\mathrm{N} 9-\mathrm{Fe} 1-\mathrm{N} 1$ & $94.52(9)$ & $\mathrm{C} 9-\mathrm{C} 8-\mathrm{H} 8 \mathrm{~A}$ & 108.4 \\
\hline $\mathrm{N} 9-\mathrm{Fe} 1-\mathrm{N} 2$ & $159.71(8)$ & $\mathrm{C} 9-\mathrm{C} 8-\mathrm{H} 8 \mathrm{~B}$ & 108.4 \\
\hline $\mathrm{N} 9-\mathrm{Fe} 1-\mathrm{N} 5$ & $84.55(8)$ & $\mathrm{C} 10-\mathrm{C} 9-\mathrm{C} 8$ & $123.0(3)$ \\
\hline N9-Fe1-N6 & $116.89(9)$ & $\mathrm{C} 10-\mathrm{C} 9-\mathrm{C} 14$ & $118.9(3)$ \\
\hline $\mathrm{N} 10-\mathrm{Fe} 1-\mathrm{N} 1$ & $116.78(7)$ & $\mathrm{C} 14-\mathrm{C} 9-\mathrm{C} 8$ & $118.0(3)$ \\
\hline $\mathrm{N} 10-\mathrm{Fe} 1-\mathrm{N} 2$ & $84.55(8)$ & $\mathrm{C} 9-\mathrm{C} 10-\mathrm{H} 10$ & 120.0 \\
\hline $\mathrm{N} 10-\mathrm{Fe} 1-\mathrm{N} 5$ & $164.17(7)$ & $\mathrm{C} 9-\mathrm{C} 10-\mathrm{C} 11$ & $120.0(3)$ \\
\hline $\mathrm{N} 10-\mathrm{Fe} 1-\mathrm{N} 6$ & $97.64(7)$ & $\mathrm{C} 11-\mathrm{C} 10-\mathrm{H} 10$ & 120.0 \\
\hline N10-Fe1-N9 & $87.58(9)$ & $\mathrm{C} 10-\mathrm{C} 11-\mathrm{H} 11$ & 120.0 \\
\hline $\mathrm{C} 4-\mathrm{N} 1-\mathrm{Fe} 1$ & $121.79(15)$ & $\mathrm{C} 12-\mathrm{C} 11-\mathrm{C} 10$ & $120.0(4)$ \\
\hline $\mathrm{C} 5-\mathrm{N} 1-\mathrm{Fe} 1$ & $119.40(16)$ & $\mathrm{C} 12-\mathrm{C} 11-\mathrm{H} 11$ & 120.0 \\
\hline $\mathrm{C} 5-\mathrm{N} 1-\mathrm{C} 4$ & $117.8(2)$ & $\mathrm{C} 11-\mathrm{C} 12-\mathrm{H} 12$ & 120.0 \\
\hline $\mathrm{N} 3-\mathrm{N} 2-\mathrm{Fe} 1$ & $137.20(15)$ & $\mathrm{C} 13-\mathrm{C} 12-\mathrm{C} 11$ & $119.9(4)$ \\
\hline N3-N2-C6 & $109.88(19)$ & $\mathrm{C} 13-\mathrm{C} 12-\mathrm{H} 12$ & 120.0 \\
\hline $\mathrm{C} 6-\mathrm{N} 2-\mathrm{Fe} 1$ & $111.96(15)$ & $\mathrm{C} 12-\mathrm{C} 13-\mathrm{H} 13$ & 119.9 \\
\hline $\mathrm{N} 2-\mathrm{N} 3-\mathrm{N} 4$ & $106.06(18)$ & $\mathrm{C} 12-\mathrm{C} 13-\mathrm{C} 14$ & $120.3(4)$ \\
\hline $\mathrm{N} 3-\mathrm{N} 4-\mathrm{C} 8$ & $119.0(2)$ & $\mathrm{C} 14-\mathrm{C} 13-\mathrm{H} 13$ & 119.9 \\
\hline $\mathrm{C} 7-\mathrm{N} 4-\mathrm{N} 3$ & $111.3(2)$ & C9-C14-H14 & 119.5 \\
\hline $\mathrm{C} 7-\mathrm{N} 4-\mathrm{C} 8$ & $129.7(2)$ & $\mathrm{C} 13-\mathrm{C} 14-\mathrm{C} 9$ & $120.9(4)$ \\
\hline $\mathrm{C} 15-\mathrm{N} 5-\mathrm{Fe} 1$ & $125.38(14)$ & $\mathrm{C} 13-\mathrm{C} 14-\mathrm{H} 14$ & 119.5 \\
\hline $\mathrm{C} 16-\mathrm{N} 5-\mathrm{Fe} 1$ & $115.78(16)$ & $\mathrm{N} 5-\mathrm{C} 15-\mathrm{C} 3$ & $112.87(19)$ \\
\hline
\end{tabular}




\begin{tabular}{|c|c|c|c|}
\hline $\mathrm{C} 16-\mathrm{N} 5-\mathrm{C} 15$ & $118.8(2)$ & $\mathrm{N} 5-\mathrm{C} 15-\mathrm{H} 15 \mathrm{~A}$ & 109.0 \\
\hline N7-N6-Fe1 & $135.01(15)$ & $\mathrm{N} 5-\mathrm{C} 15-\mathrm{H} 15 \mathrm{~B}$ & 109.0 \\
\hline $\mathrm{N} 7-\mathrm{N} 6-\mathrm{C} 17$ & $109.86(18)$ & $\mathrm{C} 3-\mathrm{C} 15-\mathrm{H} 15 \mathrm{~A}$ & 109.0 \\
\hline $\mathrm{C} 17-\mathrm{N} 6-\mathrm{Fe} 1$ & $113.90(14)$ & $\mathrm{C} 3-\mathrm{C} 15-\mathrm{H} 15 \mathrm{~B}$ & 109.0 \\
\hline $\mathrm{N} 6-\mathrm{N} 7-\mathrm{N} 8$ & $106.19(18)$ & $\mathrm{H} 15 \mathrm{~A}-\mathrm{C} 15-\mathrm{H} 15 \mathrm{~B}$ & 107.8 \\
\hline $\mathrm{N} 7-\mathrm{N} 8-\mathrm{C} 19$ & $119.86(19)$ & N5-C16-H16 & 121.5 \\
\hline $\mathrm{C} 18-\mathrm{N} 8-\mathrm{N} 7$ & $111.16(19)$ & $\mathrm{N} 5-\mathrm{C} 16-\mathrm{C} 17$ & $116.9(2)$ \\
\hline $\mathrm{C} 18-\mathrm{N} 8-\mathrm{C} 19$ & $128.89(19)$ & $\mathrm{C} 17-\mathrm{C} 16-\mathrm{H} 16$ & 121.5 \\
\hline $\mathrm{C} 26-\mathrm{N} 9-\mathrm{Fe} 1$ & $176.7(2)$ & $\mathrm{N} 6-\mathrm{C} 17-\mathrm{C} 16$ & $118.5(2)$ \\
\hline $\mathrm{C} 27-\mathrm{N} 10-\mathrm{Fe} 1$ & $165.1(2)$ & $\mathrm{N} 6-\mathrm{C} 17-\mathrm{C} 18$ & $107.5(2)$ \\
\hline $\mathrm{H} 1 \mathrm{~A}-\mathrm{C} 1-\mathrm{H} 1 \mathrm{~B}$ & 109.5 & $\mathrm{C} 18-\mathrm{C} 17-\mathrm{C} 16$ & $134.0(2)$ \\
\hline $\mathrm{H} 1 \mathrm{~A}-\mathrm{C} 1-\mathrm{H} 1 \mathrm{C}$ & 109.5 & $\mathrm{~N} 8-\mathrm{C} 18-\mathrm{C} 17$ & $105.3(2)$ \\
\hline $\mathrm{H} 1 \mathrm{~B}-\mathrm{C} 1-\mathrm{H} 1 \mathrm{C}$ & 109.5 & $\mathrm{~N} 8-\mathrm{C} 18-\mathrm{H} 18$ & 127.3 \\
\hline $\mathrm{C} 3-\mathrm{C} 1-\mathrm{H} 1 \mathrm{~A}$ & 109.5 & $\mathrm{C} 17-\mathrm{C} 18-\mathrm{H} 18$ & 127.3 \\
\hline $\mathrm{C} 3-\mathrm{C} 1-\mathrm{H} 1 \mathrm{~B}$ & 109.5 & $\mathrm{~N} 8-\mathrm{C} 19-\mathrm{H} 19 \mathrm{~A}$ & 109.0 \\
\hline $\mathrm{C} 3-\mathrm{C} 1-\mathrm{H} 1 \mathrm{C}$ & 109.5 & $\mathrm{~N} 8-\mathrm{C} 19-\mathrm{H} 19 \mathrm{~B}$ & 109.0 \\
\hline $\mathrm{H} 2 \mathrm{~A}-\mathrm{C} 2-\mathrm{H} 2 \mathrm{~B}$ & 109.5 & $\mathrm{~N} 8-\mathrm{C} 19-\mathrm{C} 20$ & $113.00(18)$ \\
\hline $\mathrm{H} 2 \mathrm{~A}-\mathrm{C} 2-\mathrm{H} 2 \mathrm{C}$ & 109.5 & $\mathrm{H} 19 \mathrm{~A}-\mathrm{C} 19-\mathrm{H} 19 \mathrm{~B}$ & 107.8 \\
\hline $\mathrm{H} 2 \mathrm{~B}-\mathrm{C} 2-\mathrm{H} 2 \mathrm{C}$ & 109.5 & $\mathrm{C} 20-\mathrm{C} 19-\mathrm{H} 19 \mathrm{~A}$ & 109.0 \\
\hline $\mathrm{C} 3-\mathrm{C} 2-\mathrm{H} 2 \mathrm{~A}$ & 109.5 & $\mathrm{C} 20-\mathrm{C} 19-\mathrm{H} 19 \mathrm{~B}$ & 109.0 \\
\hline $\mathrm{C} 3-\mathrm{C} 2-\mathrm{H} 2 \mathrm{~B}$ & 109.5 & $\mathrm{C} 21-\mathrm{C} 20-\mathrm{C} 19$ & $118.8(2)$ \\
\hline $\mathrm{C} 3-\mathrm{C} 2-\mathrm{H} 2 \mathrm{C}$ & 109.5 & $\mathrm{C} 21-\mathrm{C} 20-\mathrm{C} 25$ & $118.4(2)$ \\
\hline $\mathrm{C} 1-\mathrm{C} 3-\mathrm{C} 4$ & $106.8(2)$ & $\mathrm{C} 25-\mathrm{C} 20-\mathrm{C} 19$ & $122.7(2)$ \\
\hline $\mathrm{C} 2-\mathrm{C} 3-\mathrm{C} 1$ & $109.2(2)$ & $\mathrm{C} 20-\mathrm{C} 21-\mathrm{H} 21$ & 119.5 \\
\hline $\mathrm{C} 2-\mathrm{C} 3-\mathrm{C} 4$ & $111.5(2)$ & $\mathrm{C} 22-\mathrm{C} 21-\mathrm{C} 20$ & $121.0(2)$ \\
\hline $\mathrm{C} 2-\mathrm{C} 3-\mathrm{C} 15$ & $110.2(2)$ & $\mathrm{C} 22-\mathrm{C} 21-\mathrm{H} 21$ & 119.5 \\
\hline $\mathrm{C} 15-\mathrm{C} 3-\mathrm{C} 1$ & $107.8(2)$ & $\mathrm{C} 21-\mathrm{C} 22-\mathrm{H} 22$ & 120.1 \\
\hline $\mathrm{C} 15-\mathrm{C} 3-\mathrm{C} 4$ & $111.2(2)$ & $\mathrm{C} 21-\mathrm{C} 22-\mathrm{C} 23$ & $119.7(3)$ \\
\hline $\mathrm{N} 1-\mathrm{C} 4-\mathrm{C} 3$ & $111.39(18)$ & $\mathrm{C} 23-\mathrm{C} 22-\mathrm{H} 22$ & 120.1 \\
\hline $\mathrm{N} 1-\mathrm{C} 4-\mathrm{H} 4 \mathrm{~A}$ & 109.4 & $\mathrm{C} 22-\mathrm{C} 23-\mathrm{H} 23$ & 120.0 \\
\hline $\mathrm{N} 1-\mathrm{C} 4-\mathrm{H} 4 \mathrm{~B}$ & 109.4 & $\mathrm{C} 24-\mathrm{C} 23-\mathrm{C} 22$ & $120.0(3)$ \\
\hline $\mathrm{C} 3-\mathrm{C} 4-\mathrm{H} 4 \mathrm{~A}$ & 109.4 & $\mathrm{C} 24-\mathrm{C} 23-\mathrm{H} 23$ & 120.0 \\
\hline $\mathrm{C} 3-\mathrm{C} 4-\mathrm{H} 4 \mathrm{~B}$ & 109.4 & $\mathrm{C} 23-\mathrm{C} 24-\mathrm{H} 24$ & 119.9 \\
\hline $\mathrm{H} 4 \mathrm{~A}-\mathrm{C} 4-\mathrm{H} 4 \mathrm{~B}$ & 108.0 & $\mathrm{C} 23-\mathrm{C} 24-\mathrm{C} 25$ & $120.2(2)$ \\
\hline $\mathrm{N} 1-\mathrm{C} 5-\mathrm{H} 5$ & 121.1 & $\mathrm{C} 25-\mathrm{C} 24-\mathrm{H} 24$ & 119.9 \\
\hline $\mathrm{N} 1-\mathrm{C} 5-\mathrm{C} 6$ & $117.7(2)$ & $\mathrm{C} 20-\mathrm{C} 25-\mathrm{H} 25$ & 119.7 \\
\hline $\mathrm{C} 6-\mathrm{C} 5-\mathrm{H} 5$ & 121.1 & $\mathrm{C} 24-\mathrm{C} 25-\mathrm{C} 20$ & $120.6(2)$ \\
\hline $\mathrm{N} 2-\mathrm{C} 6-\mathrm{C} 5$ & $117.2(2)$ & $\mathrm{C} 24-\mathrm{C} 25-\mathrm{H} 25$ & 119.7 \\
\hline $\mathrm{N} 2-\mathrm{C} 6-\mathrm{C} 7$ & $107.9(2)$ & N9-C26-S1 & $179.2(3)$ \\
\hline $\mathrm{C} 7-\mathrm{C} 6-\mathrm{C} 5$ & $134.9(2)$ & $\mathrm{N} 10-\mathrm{C} 27-\mathrm{S} 2$ & $179.6(3)$ \\
\hline $\mathrm{N} 4-\mathrm{C} 7-\mathrm{C} 6$ & $104.9(2)$ & & \\
\hline $\mathrm{Fe} 1-\mathrm{N} 1-\mathrm{C} 4-\mathrm{C} 3$ & $73.0(2)$ & $\mathrm{C} 1-\mathrm{C} 3-\mathrm{C} 15-\mathrm{N} 5$ & $177.6(2)$ \\
\hline $\mathrm{Fe} 1-\mathrm{N} 1-\mathrm{C} 5-\mathrm{C} 6$ & $-0.8(3)$ & $\mathrm{C} 2-\mathrm{C} 3-\mathrm{C} 4-\mathrm{N} 1$ & $55.2(3)$ \\
\hline $\mathrm{Fe} 1-\mathrm{N} 2-\mathrm{N} 3-\mathrm{N} 4$ & $167.35(16)$ & $\mathrm{C} 2-\mathrm{C} 3-\mathrm{C} 15-\mathrm{N} 5$ & $-63.3(3)$ \\
\hline $\mathrm{Fe} 1-\mathrm{N} 2-\mathrm{C} 6-\mathrm{C} 5$ & $11.4(2)$ & $\mathrm{C} 4-\mathrm{N} 1-\mathrm{C} 5-\mathrm{C} 6$ & $167.84(19)$ \\
\hline $\mathrm{Fe} 1-\mathrm{N} 2-\mathrm{C} 6-\mathrm{C} 7$ & $-171.13(15)$ & $\mathrm{C} 4-\mathrm{C} 3-\mathrm{C} 15-\mathrm{N} 5$ & $60.8(3)$ \\
\hline $\mathrm{Fe} 1-\mathrm{N} 5-\mathrm{C} 15-\mathrm{C} 3$ & $-59.1(3)$ & $\mathrm{C} 5-\mathrm{N} 1-\mathrm{C} 4-\mathrm{C} 3$ & $-95.4(2)$ \\
\hline
\end{tabular}




$$
\begin{aligned}
& \text { Fe1-N5-C16-C17 } \\
& \text { Fe1-N6-N7-N8 } \\
& \text { Fe1-N6-C17-C16 } \\
& \text { Fe1-N6-C17-C18 } \\
& \text { N1-C5-C6-N2 } \\
& \text { N1-C5-C6-C7 } \\
& \text { N2-N3-N4-C7 } \\
& \text { N2-N3-N4-C8 } \\
& \text { N2-C6-C7-N4 } \\
& \text { N3-N2-C6-C5 } \\
& \text { N3-N2-C6-C7 } \\
& \text { N3-N4-C7-C6 } \\
& \text { N3-N4-C8-C9 } \\
& \text { N4-C8-C9-C10 } \\
& \text { N4-C8-C9-C14 } \\
& \text { N5-C16-C17-N6 } \\
& \text { N5-C16-C17-C18 } \\
& \text { N6-N7-N8-C18 } \\
& \text { N6-N7-N8-C19 } \\
& \text { N6-C17-C18-N8 } \\
& \text { N7-N6-C17-C16 } \\
& \text { N7-N6-C17-C18 } \\
& \text { N7-N8-C18-C17 } \\
& \text { N7-N8-C19-C20 } \\
& \text { N8-C19-C20-C21 } \\
& \text { N8-C19-C20-C25 } \\
& \text { C1-C3-C4-N1 }
\end{aligned}
$$

$-1.8(3)$
$166.53(16)$
$10.7(3)$
$-169.65(16)$
$-7.7(3)$
$175.7(2)$
$0.4(3)$
$178.3(2)$
$0.6(3)$
$-177.83(19)$
$-0.4(3)$
$-0.6(3)$
$103.5(3)$
$37.7(4)$
$-146.0(3)$
$-6.0(3)$
$174.4(3)$
$-0.4(3)$
$-177.12(19)$
$0.1(3)$
$180.0(2)$
$-0.4(3)$
$0.1(3)$
$74.0(3)$
$-166.2(2)$
$16.8(3)$
$174.4(2)$

$177.4(2)$

$0.0(2)$

$-79.0(3)$

$-178.2(2)$

$177.6(3)$

$-176.8(3)$

$-0.5(5)$

$-0.3(5)$

$-1.3(6)$

$2.3(6)$

$-1.5(5)$

$1.3(5)$

$175.0(2)$

$-68.1(3)$

$124.5(2)$

$179.7(3)$

$0.4(2)$

$-102.2(3)$

$176.5(2)$

$-175.6(2)$

$175.1(2)$

$0.0(4)$

$-1.9(3)$

$-1.2(4)$

$0.8(4)$

$0.8(4)$

$1.5(4)$

Hydrogen-bond geometry $\left(\AA,{ }^{\circ}\right)$

$\mathrm{Cg}$ is the centroid of the $\mathrm{C} 20-\mathrm{C} 25$ ring.

\begin{tabular}{lllll}
\hline$D-\mathrm{H} \cdots A$ & $D-\mathrm{H}$ & $\mathrm{H} \cdots A$ & $D \cdots A$ & $D-\mathrm{H} \cdots A$ \\
\hline $\mathrm{C} 18-\mathrm{H} 18 \cdots C g^{\mathrm{i}}$ & 0.93 & 2.42 & $3.330(3)$ & 167 \\
$\mathrm{C} 19-\mathrm{H} 19 A \cdots \mathrm{N} 7^{\mathrm{ii}}$ & 0.97 & 2.38 & $3.311(3)$ & 162 \\
$\mathrm{C} 21-\mathrm{H} 21 \cdots \mathrm{C} 27^{\mathrm{ii}}$ & 0.93 & 2.89 & $3.603(3)$ & 134 \\
$\mathrm{C} 7-\mathrm{H} 7 \cdots \mathrm{S} 1^{\mathrm{iii}}$ & 0.93 & 2.87 & $3.755(3)$ & 159 \\
$\mathrm{C} 4-\mathrm{H} 4 B \cdots \mathrm{N} 10^{\mathrm{iii}}$ & 0.97 & 2.69 & $3.617(3)$ & 160 \\
$\mathrm{C} 4-\mathrm{H} 4 B \cdots \mathrm{C} 27^{\mathrm{iii}}$ & 0.97 & 2.75 & $3.709(3)$ & 171 \\
\hline
\end{tabular}

Symmetry codes: (i) $-x,-y,-z+1$; (ii) $-x+1,-y,-z+1$; (iii) $-x+1,-y+1,-z$. 